\title{
Testis-sparing surgery in testicular mass: Testicular epidermoid cysts
}

\author{
Reza Mahdavi-Zafarghandi, MD; ${ }^{*}$ Behnam Shakiba, MD; ${ }^{*}$ Mojtaba Ameli, $M D^{+}$ \\ *Department of Urology, Imam Reza Hospital, Mashhad University of Medical Sciences, Mashhad, Iran; 'Department of Urology, Gonabad University of Medical Sciences, Gonabad, Iran
}

Cite as: Can Urol Assoc J 2014;8(1-2):E101-3. http://dx.doi.org/10.5489/cuaj.1588 Published online February 12, 2014.

\section{Abstract}

We present 3 patients with testicular epidermoid cysts who experienced testis-sparing surgery. These patients had a palpable painless testicular mass and underwent inguinal testicular exploration. Intraoperative frozen section revealed no evidence of malignancy and therefore enucleation of the tumour was performed. We demonstrate that careful intraoperative frozen-section examination helps to avoid unnecessary orchidectomy in testicular epidermoid cysts.

$\mathrm{T}$ esticular epidermoid cyst (TEC) was initially described by Priestley and Dockerty in $1942 .{ }^{1}$ TECs, also known as keratocysts, are rare tumours, accounting for $1 \%$ of all testicular tumours. ${ }^{2}$ Patients with TEC range in age from 3 to 77 years, with most occurring in men between 20 and $40 .{ }^{3}$ TECs present with a firm, well-circumscribed, small, solitary, painless nodule, such as testicular malignancies. TECs are benign, simple epithelial tumors which have no potential to become malignant. ${ }^{3}$ Early diagnosis is important because treating TEC is possible by enucleation of the mass and biopsy of adjacent testicular tissue to determine the presence or absence of carcinoma in situ. Testicle-preserving surgery is now favourable rather than unnecessary orchidectomy. ${ }^{4}$ We present 3 patients with testicular epidermoid cysts who had testis-sparing surgery.

\section{Case 1}

An 18-year-old male presented to our urology clinic after a 1-year history of a palpable painless left testicular mass. On physical examination, we found a well-defined, hard and non-tender mass confirmed to the upper pole of the left testis. Ultrasonography revealed a well-defined hypoechoic mass with hyperechoic centres. Tumour markers for testicu- lar cancer, including alpha-fetoprotein (AFP), beta-human chorionic gonadotropin ( $\beta$-HCG) and lactic acid dehydrogenase (LDH) levels, were normal. He underwent left inguinal testicular exploration. When we opened the tunica albuginea, a $1.5-\mathrm{cm}$ intratesticular solid lesion with calcified outer walls was enucleated. Intraoperative frozen section showed no malignant cells and microscopic histopathology confirmed a benign epidermoid cyst with stratified squamous epithelium and keratinization. He was discharged after 2 days without complications.

\section{Case 2}

A 21-year-old man was referred to the urology clinic due to a painless right testicular mass for about 6 months. He had no history of trauma or systemic diseases. On examination, we found a hard, non-tender mass in the lower pole of the right testis. Serum alpha fetoprotein (AFP), beta human chorionic gonadotropin ( $\beta$-HCG) and lactate dehydrogenase (LDH) levels were normal. Ultrasonography revealed a well-defined hypoechoic mass with a hyperechoic centre surrounded by a hyperechoic wall. He underwent a right inguinal testicular exploration. Intraoperative frozen section revealed no malignant cells. Enucleation of the intratesticular mass was performed with sparing of the testis (Fig. 1). Microscopic histopathology showed a benign epidermoid cyst. He was discharged after 2 days with no complications.

\section{Case 3}

An 18-year-old man was admitted to our hospital with the chief complaint of a painless right testicular mass. On physical examination, we found a semi-hard, non-tender mass at the inferior pole of the right testis. Tumour markers for testicular cancer (serum AFP, b-HCG and LDH) were normal. Testicular ultrasonography revealed a solid mass at the right testis with concentric rings of hypoechogenicity and hyperechogenicity. Right inguinal testicular exploration was 


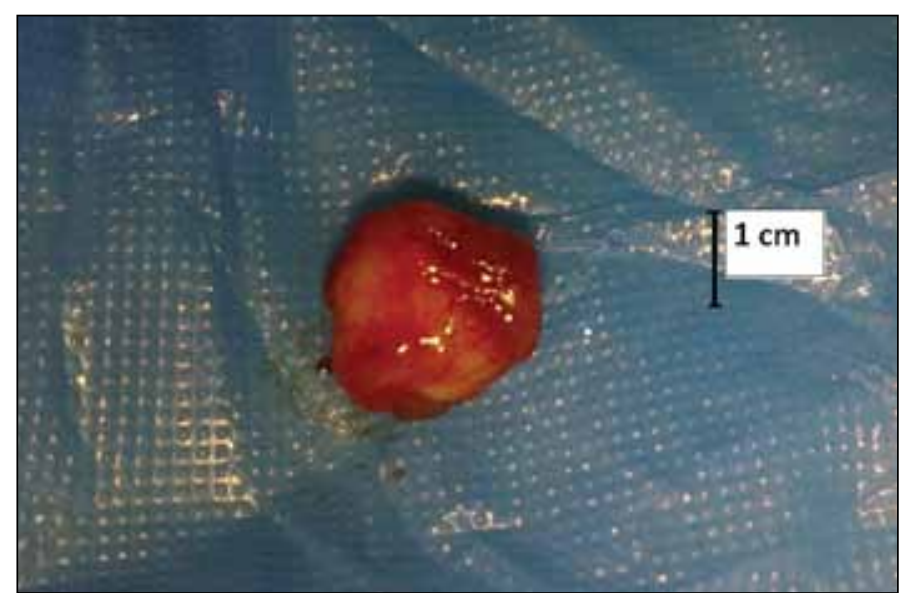

Fig. 1. Enucleated testicular epidermoid cysts.

performed and intraoperative frozen section biopsy revealed no evidence of malignancy. On opening of the tunica albuginea, we found a $2-\mathrm{cm}$ intratesticular solid lesion with calcified outer walls which were enucleated. Microscopic histopathology confirmed a benign epidermoid cyst (Fig. 2). He was discharged within 2 days without any complications.

\section{Discussion}

Intratesticular epidermoid cysts (keratocysts) are uncommon masses that appear in the second to fourth decades of life. ${ }^{5}$ TEC is a rare benign lesion, with controversial histological origins. Some studies reported that these tumours derive from germ cells. Clinically, intratesticular epidermoid cysts present as a 1 - to $3-\mathrm{cm}$ incidental smooth, firm, solitary unilateral mass. Other reports indicated multiple intratesticular epidermoid cysts in patients with Gardner syndrome, Klinefelter syndrome, cryptorchid testes and microscopic focus of primary testicular carcinoid tumour. $3,6,7$

From the clinical point of view, intratesticular epidermoid cysts are impossible to differentiate from testicular malignant tumours. In the past, radical orchidectomy was the treatment of choice for these tumours; today, however, conservative enucleation of the mass and testicle-reserving surgery are widely accepted as the best possible treatment. Therefore, it is very important to distinguish epidermal cysts from testicular malignancies. Physical examination, ultrasonography and serum tumour markers can help to make this distinction between TEC and malignancies, but the gold standard for diagnosis is a histological study. Tumour markers, such as serum $\beta$-HCG, AFP and LDH, are generally negative in TEC.

Sonographic features vary and mainly include: (1) a mass with "target" or "bull's eye" pattern, (2) a sharp mass with hyperechoic rim, (3) a circumscribed intra-testicular mass with a rim of calcification, and (4) the classic presentation of an "onion peel" appearance. On Doppler examination, no blood flow was detected in the cysts. The lack of vascularity

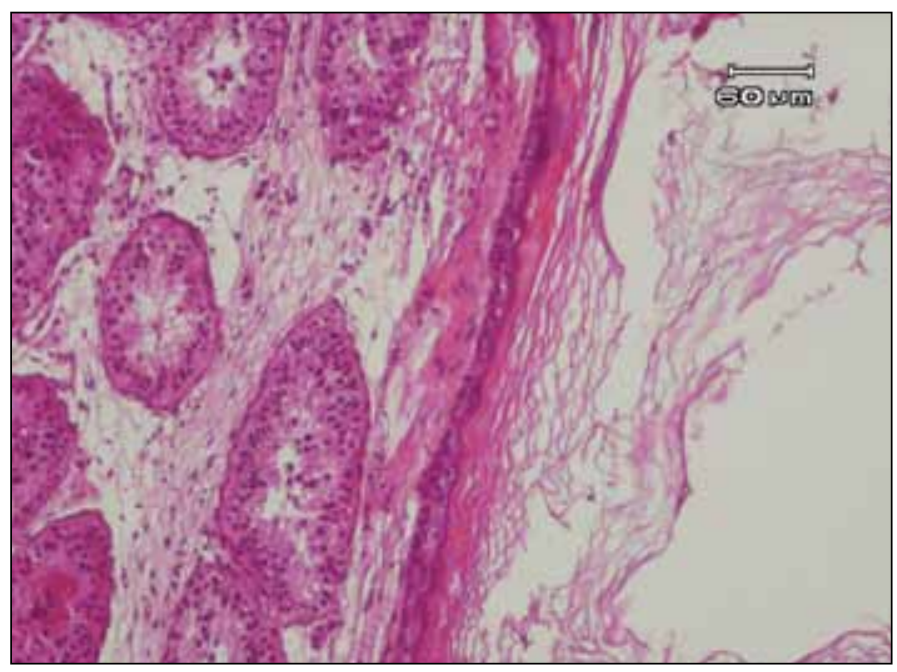

Fig. 2. Microscopic histopathology confirmed a benign epidermoid cyst.

in the cyst helps radiologists to distinguish epidermoid cysts from testicular malignant tumours. ${ }^{5,6}$

A number of studies have reported the value of magnetic resonance imaging (MRI) in the diagnosis of TEC. Based on these studies, TEC may appear at MRI as concentric rings of low and high signal intensity on T1 and T2 weighted images or a peripheral rim with low signal intensity on both $\mathrm{T} 1$ and T2 weighted images. ${ }^{3,8,9}$

The pathological findings in TEC show a keratin-filled cyst located in the normal parenchyma of the testis. The wall of the cyst is composed of fibrous tissue and squamous epithelium. There is no teratoma or other malignancies. ${ }^{10}$ Studies on the accuracy of frozen sections in differentiating benign from malignant testicular masses have equivocal results; overall, however, it seems that frozen sections have an acceptable accuracy. Several studies have reported a $94.2 \%$ positive predictive value and a $92.6 \%$ negative predictive value for frozen sections in malignancy detection. ${ }^{11,12}$

\section{Conclusion}

The presence of special radiologic features, in addition to negative tumour markers, should raise the preoperative suspicion of TEC. Based on this probable diagnosis, careful intraoperative frozen section evaluation helps urologists avoid unnecessary orchidectomy in certain cases.

Acknowledgements: The authors wish to thank Dr. Toktam Moghiman who kindly edited the manuscript.

Competing interests: Dr. Mahdavi-Zafarghandi, Dr. Shakiba and Dr. Ameli all declare no competing financial or personal interests. 
This paper has been peer-reviewed.

\section{References}

1. Omar MA, Ochai J, Natarajan C, et al. Epidermoid cyst in a solitary testis: a case for non-surgical management. Surgeon 2004;2:352-3. http://dx.doi.org/10.1016/S1479-666X(04)80036-2

2. Parlaktas BS, Köseo lu RD, Uluocak. Testis-sparing surgery for epidermoid cyst of testis in an infertile man. Erciyes Medical Journal 2005;27:75-7.

3. Venyo $A$, Kattedath $M$, Benatar $B$. Epidermoid Cyst of the testis: A case report and review of the literature. WebmedCentral Urology 2010;1:WMC001388.

4. Li WM, Su CM, Kang WY, et al. Conservative surgery for epidermoid cyst of the testis: A case report and recent literature review. JTUA 2008; 19:242-4.

5. Aneiros-Fernandez J, Arias-Santiago $S$, Cancela-Diez B, et al. Intratesticular epidermoid cyst: A rare tumor. J Clin Med Res 2010;2:281-3.

6. Tan LTH, Ong KL. Painless benign epidermoid cyst of the testis. Hong Kong J Emerg Med 2006;13:235-6.

7. Yoshida T, Takao T, Tsujimura A, et al. Testicular epidermoid cyst in Klinefelter's syndrome. Int I Urol 2006;13:478-80. http://dx.doi.org/10.1111/i.1442-2042.2006.01331.x
8. Langer JE, Ramchandani P, Siegelman ES, et al. Epidermoid cysts of the testicle: sonographic and MR Imaging features. AJR Am J Roentgennol 1999;173:1296-9. http://dx.doi.org/10.2214/ air.173.5.10541108

9. Loya AG, Said JW, Grant EG. Epidermoid cyst of the testis: Radiologic-pathologic correlation. Radiographics 2004;24:S243-6.

10. Yeh KC, Liu HY, Chou SC, et al. Organ-preserving surgery for 2 epidermoid cysts in 1 testis using ultrasonography and magnetic resonance imaging. JTUA 2004; 15:84-8.

11. Connolly SS, D'Arcy FT, Bredin HC, et al. Value of frozen section analysis with suspected testicular malignancy. Urology 2006;67:162-5. hitp://dx.doi.org/10.1016/i.urology.2005.07.041

12. Kirkham AP, Kumar $P$, Minhas $S$, et al. Targeted testicular excision biopsy: when and how should we try to avoid radical orchidectomy? Clin Radiol 2009;64:1158-65. http://dx.doi.org/10.1016/i. crad.2009.06.008

Correspondence: Dr. Behnam Shakiba, Dr. Behnam Shakiba, Department of Urology, Imam Reza Hospital, Mashhad University of Medical Sciences, Mashhad, Iran; b_shakiba@razi.tums.ac.ir 